\title{
Multianalyte Detection of Cancer Biomarkers in Human Serum Using Label-free Field Effect Transistor Biosensor
}

\author{
$\mathrm{Ke} \mathrm{Si},{ }^{1 \dagger}$ Shanshan Cheng, ${ }^{1 * \dagger}$ Sho Hideshima, ${ }^{2}$ \\ Shigeki Kuroiwa, ${ }^{2}$ Takuya Nakanishi, ${ }^{2}$ and Tetsuya Osaka ${ }^{2}$ \\ ${ }^{1}$ Department of Chemistry, Tianjin Key Laboratory of Molecular Optoelectronic Sciences, School of Science, \\ Tianjin University, Tianjin 300072, China \\ ${ }^{2}$ Smart Energy System Innovation Center (SEnSIC), \\ 513 Waseda-tsurumaki-cho, Shinjuku-ku, Tokyo 162-0041, Japan
}

(Received November 15, 2017; accepted January 22, 2018)

Keywords: field effect transistor (FET), biomarker, concentration-dependent detection, multianalyte detection

Recent statistics show that the incidence and mortality of cancer are on the rise. Among all types of cancer, lung cancer and liver cancer are the most prevalent. We have many biomarkers for these two cancers, among which the cytokeratin fragment 21-1 (CYFRA 21-1) and $\alpha$-fetoprotein (AFP) are the most common and widely used. Among the various detection methods, the field effect transistor (FET) biosensor is one of the most attractive approaches, providing a label-free, fast, and low-cost electrical detection of biomarkers with high specificity and sensitivity. In previous work, we applied biomarkers for a single cancer type. With the detection of CYFRA 21-1 and neuron-specific enolase (NSE), lung cancer was differentiated in the early stages. Compared with the early work, the biomarkers for the two cancer types, CYFRA 21-1 for lung cancer and AFP for liver cancer, were prepared in this work. The detection of CYFRA 21-1 and AFP in human serum was achieved at the same time with the limits of detection of 1 and $10 \mathrm{ng} \mathrm{mL}^{-1}$, while the cut-off values were 4 and $10 \mathrm{ng} \mathrm{mL}{ }^{-1}$. The method featured short analytical time, small sample volume, and low cost. With its good selectivity and appropriate sensitivity, clinical application will become popular in the future.

\section{Introduction}

Cancer has more than 200 distinct types associated with it, affecting the organs of people over 60. For the survival of a cancer patient, early diagnosis and treatment are crucial, so that we must develop technologies applicable to the detection of cancer in early diagnosis. ${ }^{(1-6)}$ Although much research on biosensors has been carried out in order to detect the levels of cancer biomarkers, the biomarker for a single cancer type cannot provide sufficient information for cancer diagnosis, because most cancers have more than one biomarker associated with their incidence. $^{(7-9)}$ Therefore, the multianalyte biosensor for multianalyte detection has attracted much attention as a promising tool for cancer diagnosis, with the merits of short analytical time, 
low cost, simple procedure, and small sample consumption compared with biosensors for single analytes. ${ }^{(9)}$

Many methods based on electrochemical multianalyte immunoassay have been well developed for multianalyte detection. ${ }^{(10-13)}$ For example, Wilson and Nie used a sensor consisting of an array of immunosensing electrodes for the detection of seven tumor markers, where each electrode has a different immobilized antigen. ${ }^{(14)}$ In addition, Wu et al. reported a screen-printed array for simultaneous electrochemical determination of tumor markers. ${ }^{(13)}$ However, traditional immunoassay such as enzyme-linked immunosorbent assay (ELISA) usually requires a labelling process, resulting in the lack of rapidity and simplicity. ${ }^{(8,9,15,16)}$ In comparison, the biosensor based on a field effect device has attracted great attention for labelfree detection of biomolecules. ${ }^{(9,15-25)}$ The other label-free methods such as surface plasmon resonance (SPR) ${ }^{(26,27)}$ and quartz crystal microbalance $(\mathrm{QCM})^{(28,29)}$ require lengthy steps and complex handling. In addition, immunochromatography and chromatography are quick and simple, however, they require expensive instrumentation. However, field effect transistors (FETs) have emerged as an important new technique since they do not require any labeling ${ }^{(24)}$ and allow highly sensitive detection. Moreover, these devices provide numerous potential advantages including small size and light weight, the prospect of low-cost mass production, and the possibility of on-chip integration of arrays. ${ }^{(9,16)}$ Some multianalyte detection methods based on FETs have been proposed over the past decade. ${ }^{(15,30-32)}$ Zheng et al. reported work on multiplexed detection of several cancer markers with a silicon-nanowire (SiNW) array. ${ }^{(15)}$ Jia et al. developed a multitumor marker detection system based on a light addressable potentiometric sensor (LAPS). ${ }^{(32)}$ The development of biosensor assays based on field effect devices is significant in multianalyte determination of biomolecules.

We are committed to developing biosensors based on FETs for the detection of multianalyte cancer biomarkers, particularly in biological samples (e.g., serum). Previously, Cheng et al. proposed an antibody-based multianalyte FET biosensor for detecting cytokeratin fragment 21-1 (CYFRA 21-1) and neuron-specific enolase (NSE), which result in lung cancer, providing a step towards the realization of the array sensor. ${ }^{(24)}$ The transistor not only has selective identification, but can also be used for simultaneous detection of multiple targets, and it can also be a diagnosis method for lung cancer.

In this work, we carried out some further study. With biomarkers for multianalyte detection of two cancer types, CYFRA 21-1 and $\alpha$-fetoprotein (AFP) were each detected using our fabricated antibody-based FET biosensor, showing the potential for application to cancer diagnosis. In the device, monoclonal antibody of CYFRA 21-1 and AFP were immobilized on two separate FETs integrated on one chip [Fig. 1(c)], which was characterized by atomic force microscopy (AFM). Furthermore, we carried out simultaneous concentration-dependent detections of CYFRA 21-1 and AFP in human serum to perform quantitative analysis that can satisfy the cut-off value and be used for clinic application. We also compared the difference between the single-analyte biosensor and the multianalyte biosensor, not only was the persuasiveness of the experimental data enhanced, but also, the method can satisfy the clinical criteria. In a word, we are not limited to detecting biomarkers for a single cancer type in this work. 


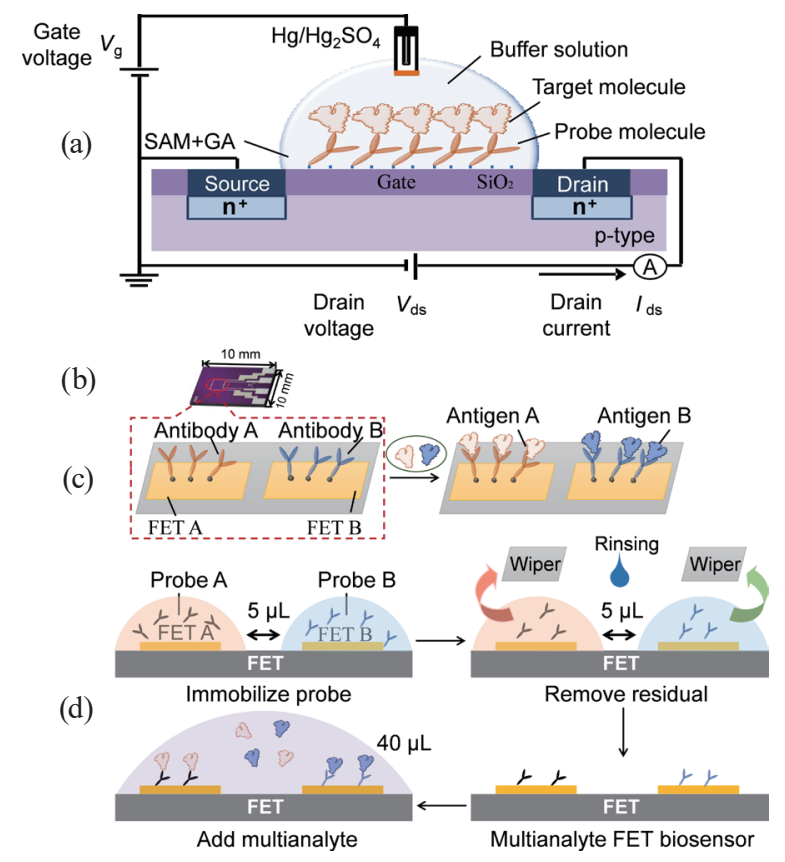

Fig. 1. (Color online) Multianalyte FET biosensor. (a) Schematic illustration of antibody-immobilized FET for cancer biomarker detection. The reference electrode was $\mathrm{Hg} / \mathrm{Hg}_{2} \mathrm{SO}_{4}$. (b) Optical pattern of FET chip. (c) Schematic of two integrated FETs, where the gates were immobilized with different antibody receptors (A, red; B, blue). (d) Schematic illustration of sensing area and solution dosage. (The amount of immobilized antibody was 5 $\mu \mathrm{L}$. The amount of multiple target was $40 \mu \mathrm{L}$.)

Our intentions were as follows: (1) firstly, multianalyte detection of CYFRA 21-1 and AFP in human serum for the diagnosis of two different cancer types, not being limited to the detection of a single cancer type; (2) then, concentration-dependent detection of CYFRA 21-1 and AFP for quantitative analysis at the same time in human serum; (3) further, the limits of detection about CYFRA 21-1 and AFP in human serum is lower than or equal to the cut-off value of clinical application; (4) finally, AFM observation of the surface morphology of the FET biosensor and presentation of a new fabrication method for the future array sensors.

\section{Materials and Methods}

\subsection{Reagents and chemicals}

The antigen, human CYFRA 21-1, was obtained from Acris Antibodies Inc. (San Diego, CA, USA). The antigen, human AFP, was purchased from MP Biomedicals, LLC (Santa Ana, CA., USA). Monoclonal anti-CYFRA 21-1 antibody (mouse) was acquired from Gene Tex Inc. (Irvine, CA, USA). Monoclonal anti-AFP antibody (human) was gained from Nippon Bio-test Laboratories Inc. (Tokyo, Japan). Bovine serum albumin (BSA) was purchased from Jackson ImmunoResearch Laboratories Inc. (West Grove, PA, USA). 3-aminopropyltriethoxysilane (APTES) and human serum were stocked from Sigma-Aldrich Inc. (St. Louis, MO, USA). 
All other chemicals were obtained from Kanto Chemical Co. Inc. (Tokyo, Japan). Phosphate buffer solution (PBS) $(\mathrm{pH}=7.4)$ was prepared in the laboratory using $137 \mathrm{mM} \mathrm{NaCl}, 8.1 \mathrm{mM}$ $\mathrm{Na}_{2} \mathrm{HPO}_{4} \cdot 12 \mathrm{H}_{2} \mathrm{O}, 2.7 \mathrm{mM} \mathrm{KCl}$, and $1.5 \mathrm{mM} \mathrm{KH}_{2} \mathrm{PO}_{4}$. Antibody was diluted in $1 \times \mathrm{PBS}$ and antigen was diluted in human serum. The proteins were used without further purification.

\subsection{Apparatus}

The sensing area is shown in Fig. 1(d). The gate voltage $\left(V_{g}\right)$-drain current $\left(I_{d s}\right)$ characteristics were examined using a semiconductor parameter analyzer (2612A, Keithley Instruments Inc.; Cleveland, OH, USA) before and after antigen-antibody reaction in the dark by sweeping $V_{g}$ from -3 to $0.5 \mathrm{~V}$ with a $0.1 \mathrm{~V}$ drain voltage. $\Delta V_{g}$, the calculated threshold voltage shift, will be discussed as an FET response. All measurements were made with $0.01 \times \mathrm{PBS}(\mathrm{pH}$ = 7.4) at room temperature. $\mathrm{Hg} / \mathrm{Hg}_{2} \mathrm{SO}_{4}$ was used as a reference electrode. In this work, we use the AFM (Shimadzu Co., SPM-9600) to obtain topographic images of the FET gate surface in the dynamic mode.

\subsection{Preparation of antibody-immobilized FET biosensor for multianalytes with BSA blocking}

The FET chip consists of two transistors [Figs. 1(b) and 1(c)]. Niwa et al. have developed $\mathrm{SiO}_{2}$-gate FETs with self-assembled monolayers (SAMs). ${ }^{(21)}$ In addition, APTES SAM and the cross-linker glutaraldehyde (GA) undergo a modification process. Then, antibodies are allowed to react with the activated GA-modified FETs at room temperature for $1 \mathrm{~h}$. It can be seen from Fig. 1(c) that anti-CYFRA 21-1 antibody $\left(50 \mu \mathrm{g} \mathrm{mL} \mathrm{m}^{-1}, 5 \mu \mathrm{L}\right)$ and anti-AFP antibody $(50 \mu \mathrm{g}$ $\mathrm{mL}^{-1}, 5 \mu \mathrm{L}$ ) were deposited on each gate side by side in the FET biosensor for multianalytes. Furthermore, we used $1.0 \mathrm{~mL}$ of $0.01 \times$ PBS to rinse the residue on probe-molecule-immobilized FET five times. At last, the antibody-immobilized surface was allowed to react with BSA (1 $\mathrm{wt} \%$ ) for $60 \mathrm{~min}$ to avoid nonspecific adsorption onto the FET gate surface.

\section{Results and Discussion}

\subsection{Multianalyte detection using cancer biomarkers in human serum}

Label-free detection of biomarkers in real biological samples, such as serum, plasma, urine, and whole blood, is highly desirable as well as more practical from the viewpoint of care. Hence, we examined our multianalyte FET biosensor for the detection of two useful cancer biomarkers in human serum, not limited to the detection of a single analyte cancer type. A lung cancer biomarker, CYFRA 21-1, and a liver cancer biomarker, AFP, were selected as the target analytes. Since the nonspecific adsorption of other protein(s) in human serum may affect the sensitivity of biosensors, we introduced BSA, a well-known blocking agent, to help reduce such nonspecific binding. Anti-CYFRA 21-1 antibody and anti-AFP antibody were immobilized on the surface of each FET gate insulator separately [Fig. 1(c)] to capture the target analytes 
before BSA blocking. First of all, we analyzed the response of the BSA-blocked antibodyimmobilized multianalyte FET biosensor to a mixture of CYFRA 21-1 and AFP (multianalytes) in human serum. As shown in Fig. 2, a negligible response (i.e., $1.7 \pm 2.8 \mathrm{mV}$ for anti-CYFRA 21-1 antibody-immobilized gate; $1.8 \pm 3.5 \mathrm{mV}$ for anti-AFP antibody-immobilized gate) was acquired when the chip was exposed to analyte-free human serum (blank). This result also indicates that the nonspecific adsorption of other protein(s) in human serum was minimal. Then, after the addition of a mixture of $1 \mathrm{ng} \mathrm{mL}^{-1}$ CYFRA 21-1 and $10 \mathrm{ng} \mathrm{mL}^{-1}$ AFP in human serum, a response (i.e., $10.6 \pm 2.3 \mathrm{mV}$ for anti-CYFRA 21-1 antibody-immobilized gate; 11.7 $\pm 3.1 \mathrm{mV}$ for anti-AFP antibody-immobilized gate) was obtained from each FET gate. As expected from their isoelectric point [pI] (i.e., 5.2 for CYFRA 21-1; ${ }^{(33)} 4.9$ for $\mathrm{AFP}^{(34)}$ ) and $\mathrm{pH}$ $=7.4$ under the present experimental conditions, the positive $\Delta V_{g}$ values for CYFRA 21-1 and AFP were attributed to the intrinsic negative charges of CYFRA 21-1 and AFP. Compared with the magnitude of $\Delta V_{g}$ observed after the addition of analyte-free human serum, the magnitude of $\Delta V_{g}$ observed upon the incubation of $1 \mathrm{ng} \mathrm{mL}^{-1}$ CYFRA 21-1 and $10 \mathrm{ng} \mathrm{mL}^{-1}$ AFP was significant. In other words, the multianalyte FET biosensor successfully detected CYFRA 21-1 and AFP in human serum at the low levels of 1 and $10 \mathrm{ng} \mathrm{mL}^{-1}$, respectively, which meet the cut-off values for normal levels (i.e., $4 \mathrm{ng} \mathrm{mL}{ }^{-1}$ for CYFRA 21-1; ${ }^{(35)} 10 \mathrm{ng} \mathrm{mL}^{-1}$ for $\mathrm{AFP}^{(36)}$ ). The results illustrate that this biosensor has potential for the practical detection of cancer biomarkers in serum samples for the clinical diagnosis of cancer.

\subsection{Concentration-dependent detection of cancer biomarkers using the FET biosensor for multianalytes in human serum}

Next, we quantitatively detected CYFRA 21-1 [Fig. 3(a)] and AFP [Fig. 3(b)] using the BSA-blocked antibody-immobilized FET biosensor for multianalytes in human serum. We detected the multianalytes contained in human serum: CYFRA 21-1 at varying concentrations ranging from 1 to $100 \mathrm{ng} \mathrm{mL}^{-1}$ [Fig. 3(a), red diamonds] and $10 \mathrm{ng} \mathrm{mL}^{-1}$ AFP [Fig. 3(a), yellow squares]. We can see that the magnitude of $\Delta V_{g}$ obtained from the anti-CYFRA 21-1 antibodyimmobilized gate [Fig. 3(a), red diamonds] gradually increased as CYFRA 21-1 concentration increased in the above range. In addition, the response to human serum containing $10 \mathrm{ng} \mathrm{mL}^{-1}$ AFP (blank) was $2.2 \pm 2.9 \mathrm{mV}$, which is similar to the response to human serum without any

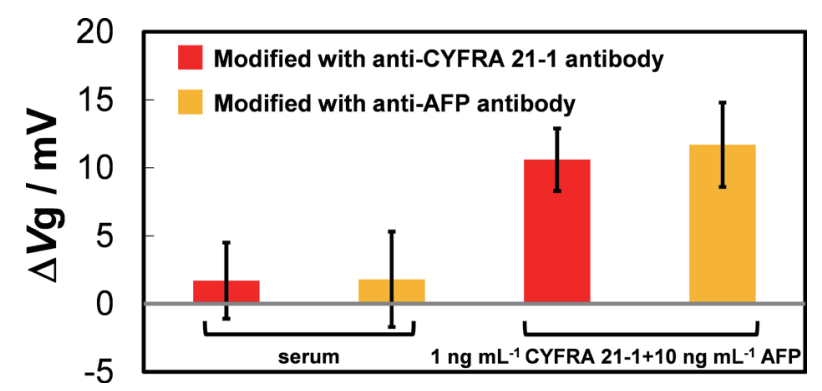

Fig. 2. (Color online) Detection of biomarkers for multianalyte cancer types (mixture of $1 \mathrm{ng} \mathrm{mL} \mathrm{m}^{-1}$ CYFRA 21-1 and $\left.10 \mathrm{ng} \mathrm{mL} \mathrm{m}^{-1} \mathrm{AFP}\right)$. The standard deviation is shown by the error bars $(n=3)$. 


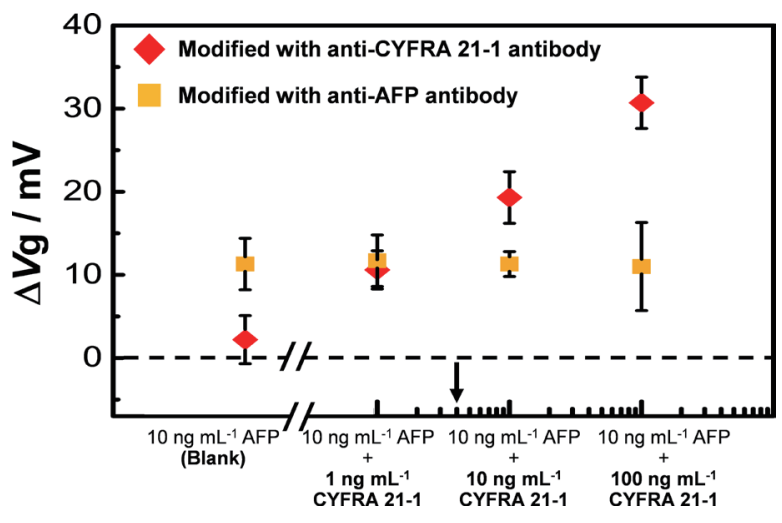

(a)

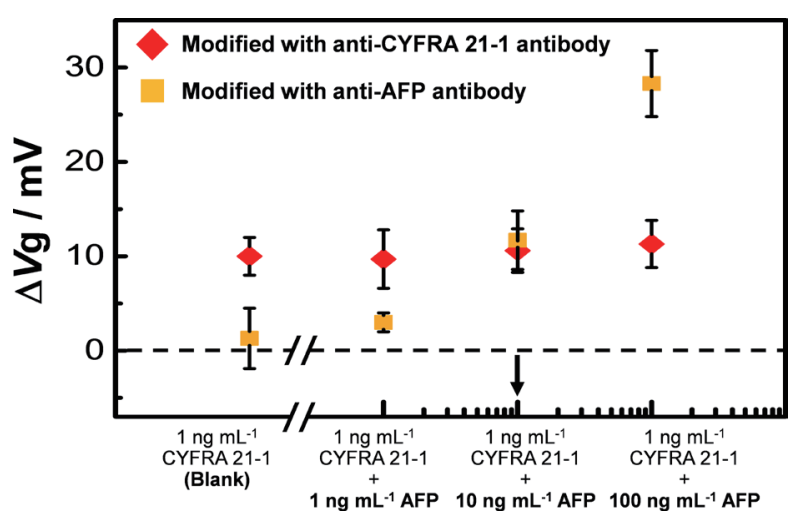

(b)

Fig. 3. (Color online) Concentration-dependent detection of (a) CYFRA 21-1 and (b) AFP using BSA-blocked FET biosensor for multianalytes (a) $10 \mathrm{ng} \mathrm{mL}^{-1}$ AFP and (b) $1 \mathrm{ng} \mathrm{mL} \mathrm{mL}^{-1}$ CYFRA 21-1. The cut-off value (4 $\mathrm{ng} \mathrm{mL}^{-1}$ for CYFRA 21-1 and $10 \mathrm{ng} \mathrm{mL}^{-1}$ for AFP) was indicated by arrows. The standard deviation is shown by the error bars $(n=3)$.

analyte (i.e., blank, $1.7 \pm 2.8 \mathrm{mV}$, see Fig. 2). Thus, compared with the blank, the detection limit for CYFRA 21-1 using the multianalyte FET biosensor in human serum was $1 \mathrm{ng} \mathrm{mL}-1$ whether AFP exists or not. On the other hand, similar magnitudes of $\Delta V_{g}$ were obtained from the anti-AFP antibody-immobilized gate [Fig. 3(a), yellow squares], showing that the effect on the existence of CYFRA 21-1 was minimal. Hence, this FET biosensor for multianalytes shows good selectivity for multianalyte detection. Similarly, as shown in Fig. 3(b), AFP at concentrations ranging from 1 to $100 \mathrm{ng} \mathrm{mL}^{-1}$ was quantitatively detected even with the existence of $1 \mathrm{ng} \mathrm{mL}^{-1}$ CYFRA 21-1 in human serum. Compared with the blank [i.e., $1.3 \pm 3.2$ $\mathrm{mV}$ for human serum containing $1 \mathrm{ng} \mathrm{mL}{ }^{-1}$ CYFRA 21-1, see Fig. 3(b); $1.8 \pm 3.5 \mathrm{mV}$ for human serum without any analyte, see Fig. 2], the detection limit of AFP in human serum was found to be $10 \mathrm{ng} \mathrm{mL}{ }^{-1}$. We could say that our biosensor achieved the quantitative detection of CYFRA 21-1 and AFP from a multianalyte sample at the low concentrations of 1 and $10 \mathrm{ng} \mathrm{mL}{ }^{-1}$ in human serum, respectively, which meets the clinical cut-off point. ${ }^{(35,36)}$ The biosensor will be more effective for the future study of cancer biomarkers in clinical and personal health care.

It should be noted that the $\Delta V_{g}$ value for CYFRA 21-1 tends to be larger than that for AFP at the same mass concentrations. Their molecular weights may explain why CYFRA 21-1 has a greater molar concentration than AFP (i.e., $40 \mathrm{kDa}$ for CYFRA $21-1 ;^{(33)} 70 \mathrm{kDa}$ for $\mathrm{AFP}^{(37)}$ ). Moreover, the second reason may be the different sizes of CYFRA 21-1 (estimated to be equivalent to a sphere with a diameter of $\left.4.5 \mathrm{~nm}^{(33)}\right)$ and AFP $\left(5 \times 5 \times 5 \mathrm{~nm}^{3(38)}\right)$. In addition, the adsorption of the AFP molecules onto the antibody-immobilized surface might be limited by steric hindrance compared with the smaller CYFRA 21-1, resulting in a decrease in the intensity of effective signals. On the other hand, under the condition of the same Debye length $(7.5 \mathrm{~nm}$ in $0.01 \times \mathrm{PBS}^{(25)}$ ), the binding event between antibody and AFP might occur outside the chargedetectable region, while the adsorbed CYFRA 21-1 is expected to be inside the Debye length, which leads to a difference in the magnitude of the FET response. 


\subsection{Comparison of sensor performance between FET biosensors for multianalytes and single analyte}

In order to confirm the sensor performance of the multianalyte FET biosensor, we compare the FET biosensor for multianalyte cancer types with that for single-analyte cancer types. The results are shown in Table 1. The FET biosensor for multianalytes has the same sensitivity as that for single analytes, for which the detection limits of CYFRA 21-1 $\left(1 \mathrm{ng} \mathrm{mL}^{-1}\right)$ and AFP $\left(10 \mathrm{ng} \mathrm{mL}^{-1}\right)$ were the same. It is possible that the immobilization conditions of the two types of sensor were the same, and the probe molecules were well immobilized on each sensing area of the multianalyte sensor after controlling the immobilization process. In a word, the FET biosensor for multianalytes shows good selectivity for multiplexed detection.

Then, we use the AFM to prove the previous assumption by observing the surface morphology of the FET biosensor for multianalytes and compare it with that of the biosensor for single analytes. As can be seen in Fig. 4. The similarity of the AFM images suggests that

Table 1

FET response for different types of FET biosensor.

\begin{tabular}{ccccr}
\hline $\begin{array}{c}\text { Concentration } \\
\left.(\mathrm{ng} \mathrm{mL})^{-1}\right)\end{array}$ & \multicolumn{2}{c}{ CYFRA 21-1 } & \multicolumn{2}{c}{ AFP } \\
\cline { 2 - 5 } & $\Delta V_{g} / \mathrm{mV}^{\mathrm{a}}$ & $\Delta V_{g} / \mathrm{mV}^{\mathrm{b}}$ & $\Delta V_{g} / \mathrm{mV}^{\mathrm{c}}$ & $\Delta V_{g} / \mathrm{mV}^{\mathrm{d}}$ \\
\hline 1 & $10.8 \pm 2.7$ & $10.6 \pm 2.3$ & - & $3.0 \pm 1.0$ \\
10 & $15.6 \pm 1.0$ & $19.3 \pm 3.1$ & $14.0 \pm 4.0$ & $11.7 \pm 3.1$ \\
100 & $22.0 \pm 5.8$ & $30.7 \pm 3.1$ & $36.0 \pm 3.0$ & $28.3 \pm 3.5$
\end{tabular}

a. FET response for CYFRA 21-1 obtained using FET biosensor for single analyte.

b. FET response for CYFRA 21-1 obtained using FET biosensor for multianalytes.

c. FET response for AFP obtained using FET biosensor for single analyte.

d. FET response for AFP obtained using FET biosensor for multianalytes.

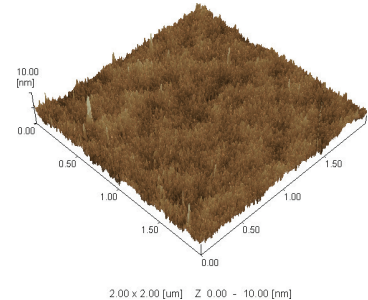

(a)

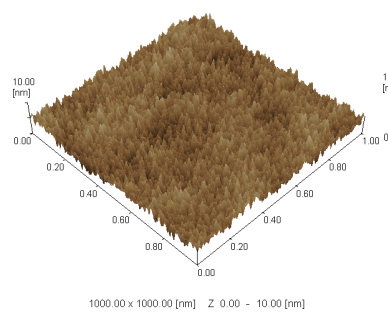

(e)

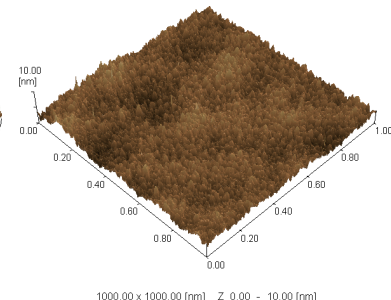

(b)

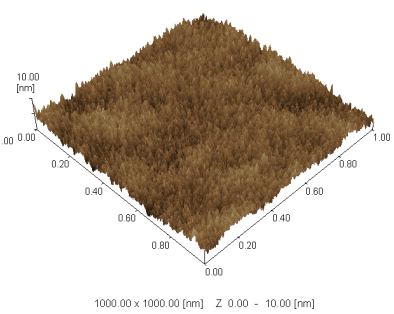

(f)

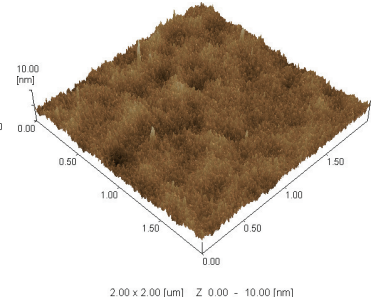

(c)

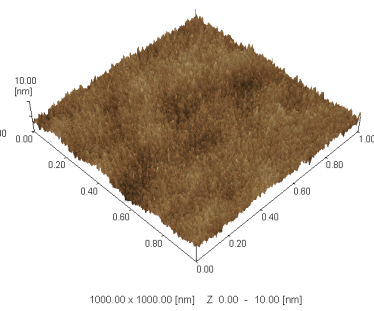

(g)

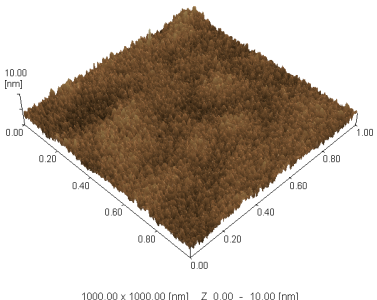

(d)

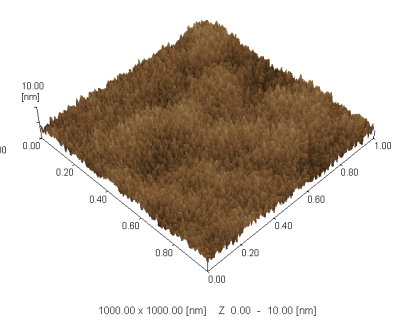

(h)

Fig. 4. (Color online) AFM images of surfaces of anti-CYFRA 21-1 antibody-immobilized FET biosensor for (a) single analyte and (b) multianalytes. BSA-blocked anti-CYFRA 21-1 antibody-immobilized FET biosensor for (c) single analyte and (d) multianalytes. Anti-AFP antibody-immobilized FET biosensor for (e) single analyte and (f) multianalytes. BSA-blocked anti-AFP antibody-immobilized FET biosensor for (g) single analyte and (h) multianalytes. The roughness $(R q)$ is (a) 0.934 (b) 0.841 (c) 0.896 (d) 0.812 (e) 1.146 (f) 0.974 (g) 1.056 , and (h) 0.936 . $Z$ range $=10 \mathrm{~nm}$. 
the immobilization of antibody onto each FET gate surface is the same in the multianalyte FET biosensor and the single-analyte FET biosensor. The decrease in $R_{q}$ (roughness) after the addition of BSA indicated that BSA molecules filled the spaces not occupied by the immobilized antibody. This demonstrates that the two types of biosensors have the same surface condition that results in the same capability of biomarker detection. With advantages such as short analytical time, small sample volume, and simple process, the performance of the FET biosensor for multianalytes is comparable to that of the FET biosensor for single analytes.

\section{Conclusions}

Two antibody types were integrated on the same FET chip as probe molecules to develop a label-free multianalyte biosensor based on FET for the detection of cancer biomarkers. It was demonstrated that our label-free, multiplexed, quantitative, sensitive sensor can be used for the detection of CYFRA 21-1 and AFP in human serum, and for the diagnosis of two different cancer types. Furthermore, the concentration ranges of CYFRA 21-1 and AFP were 1-100 ng $\mathrm{mL}^{-1}$ with the detection limits of 1 and $10 \mathrm{ng} \mathrm{mL}^{-1}$, respectively, which indicated potential for a practical method of clinical detection using serum samples. Moreover, the proposed biosensor offers an alternative to the label-free multianalyte quantitative detection of biomarkers, providing a step towards the realization of sensor arrays for use in the evaluation of multiple markers in serum. In this work, we not only determined the detection limits of CYFRA 21-1 and AFP accurately, but also performed some quantitative analysis, which was not accomplished in the previous study. Last but not least, the probability of error, as both false negative and false positive results, will be reduced by taking simultaneously exclusive biomolecule data.

\section{Acknowledgments}

The financial support from the National Natural Science Foundation of China (grant number 21705116), the Ministry of Science and Technology of China (grant numbers 2016YFB0401100, 2013CB933403, and 2013CB933504), the National Natural Science Foundation of China (grant numbers 51633006, 91222203, 91233205, and 91433115), and the Chinese Academy of Sciences (grant number XDB12030300) is gratefully acknowledged.

\section{References}

1 J. A. Ludwig and J. N. Weinstein: Nat. Rev. Cancer 5 (2005) 845.

2 M. Devillers, L. Ahmad, H. Korri-Youssoufi, and L. Salmon: Biosens. Bioelectron. 96 (2017) 178.

3 T. Liu, X. Zhang, S. Gao, F. Jing, Y. Yang, L. Du, G. Zheng, P. Li, C. Li, and C. Wang: Oncotarget 7 (2016) 85551.

4 I. E. Tothill: Semin. Cell Dev. Biol. 20 (2009) 55.

5 B. E. Rapp, F. J. Gruhl, and K. Laenge: Anal. Bioanal.Chem. 398 (2010) 2403.

6 J. Li, S. Li, and C. F. Yang: Electroanalysis 24 (2012) 2213.

7 J. D. Wulfkuhle, L. A. Liotta, and E. F. Petricoin: Nat. Rev. Cancer 3 (2003) 267.

8 J. Lin, Z. Wei, and C. Mao: Biosens. Bioelectron. 29 (2011) 40.

9 A. Poghossian and M. J. Schoening: Electroanalysis 26 (2014) 1197.

10 M. E. Meyerhoff, C. Duan, and M. Meusel: Clin. Chem. 41 (1995) 1378. 
11 J. Lin, H. Zhang, and M. Shao: Acta Chim. Sinica 72 (2014) 241.

12 Y. Fang, X. Huang, Q. Zeng, and L. Wang: Biosens. Bioelectron. 73 (2015) 71.

13 J. Wu, Z. Zhang, Z. Fu, and H. Ju: Biosens. Bioelectron. 23 (2007) 114.

14 M. S. Wilson and W. Nie: Anal. Chem. 78 (2006) 6476.

15 G. F. Zheng, F. Patolsky, Y. Cui, W. U. Wang, and C. M. Lieber: Nat. Biotechnol. 23 (2005) 1294.

16 M. J. Schoning and A. Poghossian: Analyst 127 (2002) 1137.

17 D. Singh, S. Pandey, K. Nigam, D. Sharma, D. S. Yadav, and P. Kondekar: IEEE Trans. Electron Devices 64 (2017) 271.

18 T. Sakata and Y. Miyahara: Biosens. Bioelectron. 21 (2005) 827.

19 M. Magliulo, D. De Tullio, I. Vikholm-Lundin, W. M. Albers, T. Munter, K. Manoli, G. Palazzo, and L. Torsi: Anal. Bioanal. Chem. 408 (2016) 3943.

20 T. Goda and Y. Miyahara: Biosens. Bioelectron. 45 (2013) 89.

21 D. Niwa, K. Omichi, N. Motohashi, T. Homma, and T. Osaka: Sens. Actuators, B 108 (2005) 721.

22 S. Hideshima, R. Sato, S. Inoue, S. Kuroiwa, and T. Osaka: Sens. Actuators, B 161 (2012) 146.

23 S. Hideshima, S. Kuroiwa, M. Kimura, S. Cheng, and T. Osaka: Electrochim. Acta 110 (2013) 146.

24 S. Cheng, S. Hideshima, S. Kuroiwa, T. Nakanishi, and T. Osaka: Sens. Actuators, B 212 (2015) 329.

25 S. Cheng, K. Hotani, S. Hideshima, S. Kuroiwa, T. Nakanishi, M. Hashimoto, Y. Mori, and T. Osaka: Materials 7 (2014) 2490.

26 J. Homola, S. S. Yee, and G. Gauglitz: Sens. Actuators, B 54 (1999) 3.

27 J. Homola, H. Vaisocherova, J. Dostalek, and M. Piliarik: Methods 37 (2005) 26.

28 A. Janshoff, H. J. Galla, and C. Steinem: Angew. Chem. Int. Ed. 39 (2000) 4004.

29 Y. Luo, M. Chen, Q. Wen, M. Zhao, B. Zhang, X. Li, F. Wang, Q. Huang, C. Yao, T. Jiang, G. Cai, and W. Fu: Clin. Chem. 52 (2006) 2273.

30 F. Patolsky, B. P. Timko, G. Zheng, and C. M. Lieber: MRS Bull. 32 (2007) 142.

31 A. Kim, C. S. Ah, C. W. Park, J.-H. Yang, T. Kim, C.-G. Ahn, S. H. Park, and G. Y. Sung: Biosens. Bioelectron. 25 (2010) 1767.

32 Y.-F. Jia, C.-Y. Gao, J. He, D.-F. Feng, K.-L. Xing, M. Wu, Y. Liu, W.-S. Cai, and X.-Z. Feng: Analyst 137 (2012) 3806.

33 A. He, T.-C. Liu, Z.-N. Dong, Z.-Q. Ren, J.-Y. Hou, M. Li, and Y.-S. Wu: J. Clin. Lab. Anal. 27 (2013) 277.

34 L. J. Burditt, M. M. Johnson, P. J. Johnson, and R. Williams: Cancer 74 (1994) 25.

35 X.-Y. Chu, X.-B. Hou, W.-A. Song, Z.-Q. Xue, B. Wang, and L.-B. Zhang: Cancer Biol. Ther. 11 (2011) 995.

36 J. B. Lopez: Clin. Biochem. Rev. 26 (2005) 65.

37 M. F. Yuen and C. L. Lai: Best Pract. Res. Clin. Gastroenterol. 19 (2005) 91.

38 X. Huang and J. Ren: Anal. Chim. Acta 686 (2011) 115. 